\title{
Application of nanocarbon negative imaging technology in surgery for secondary hyperparathyroidism
}

\author{
Yu Wu ${ }^{1 \#}$, Ying Liu ${ }^{2 \#}$, Tao Huang ${ }^{1 \#}$, Yasu Jiang ${ }^{3}$, Hua Wang ${ }^{1}$, Zhixian $\mathrm{He}^{1}$ \\ ${ }^{1}$ Department of General Surgery, Affiliated Hospital of Nantong University, Nantong, China; ${ }^{2}$ Department of General Surgery, Nantong Geriatric \\ Rehabilitation Hospital, Nantong, China; ${ }^{3}$ Department of General Surgery, the Second Affiliated Hospital of Nantong University, Nantong, China \\ Contributions: (I) Conception and design: Y Wu, Z He; (II) Administrative support: H Wang; (III) Provision of study materials or patients: Y Wu, \\ Y Liu; (IV) Collection and assembly of data: Y Jiang; (V) Data analysis and interpretation: Y Wu; (VI) Manuscript writing: All authors; (VII) Final \\ approval of manuscript: All authors. \\ \#These authors contributed equally to this work. \\ Correspondence to: Zhixian He. Department of General Surgery, Affiliated Hospital of Nantong University, No.20, Xisi Road, Nantong 226000, \\ China. Email: hezhixiangs@sina.com.
}

\begin{abstract}
Background: Our objective is to evaluate the application values and effects of nanocarbon negative imaging technology in surgery for patients with the fifth stage of chronic kidney disease complicated with secondary hyperparathyroidism (SHPT).

Methods: Eighty-nine patients with SHPT in the fifth stage of chronic kidney disease admitted to the Department of Thyroid and Breast Surgery at the Affiliated Hospital of Nantong University between January 2018 and August 2020 were selected. All patients underwent total parathyroidectomy (tPTX) and were randomly divided into a group receiving nanocarbon (observation group; group A) and a control group (group B). Patients were followed up for 6 months after surgery and several observation indexes were compared and analyzed.

Results: Compared with the control group, the parathyroid glands in the observation group treated with nanocarbon were more clearly exposed, and better performances were seen in the operation time, blood loss, and recovery rate of bone pain $(\mathrm{P}<0.05)$. The postoperative follow-up blood intact parathyroid hormone level (iPTH) and recurrence rate control were also improved in the observation group and the differences were statistically significant $(\mathrm{P}<0.05)$.

Conclusions: In the fifth stage of chronic kidney disease with SHPT, the application of nanocarbon negative imaging technology can significantly reduce the recurrence rate of hyperparathyroidism, improve the surgical effect, and improve the long-term quality of life and survival rate of patients.
\end{abstract}

Keywords: Nanocarbon; secondary hyperparathyroidism (SHPT); total parathyroidectomy (tPTX)

Submitted May 20, 2021. Accepted for publication Aug 19, 2021.

doi: $10.21037 / g s-21-385$

View this article at: https://dx.doi.org/10.21037/gs-21-385

\section{Introduction}

Secondary hyperparathyroidism (SHPT) is a common complication in patients with chronic kidney disease stage 5 (CKD5), and has a significant impact on the quality of life and survival time of patients $(1,2)$. A longterm increase in the intact parathyroid hormone level (iPTH) and disturbances in blood calcium and phosphorus metabolism will lead to ectopic calcium deposition in the skin, cardiovascular, and other tissue, and brings about a series of problems such as poor quality of life, poor quality of sleep, a depressed mental state, and physical discomfort $(3,4)$. More than $75 \%$ of patients have cardiac hypertrophy and left ventricular dysfunction, and more than $50 \%$ of CKD5 patients die because of cardiovascular events (5). While medical treatment may improve symptoms for a limited time, with the extension of the disease timeline, 
parathyroid hyperplasia is seen to be mainly adenomatous hyperplasia and nodular hyperplasia, parathyroid cells are mainly monoclonal and the proliferation speed is fast, and calcium-sensitive and vitamin D active drugs are gradually ineffective, resulting in parathyroidectomy as the final treatment option (6).

The continuous development and application of new technology in thyroid surgery have promoted the rapid progress of thyroid surgery. New technology in thyroid surgery has developed rapidly. With the application of neural monitoring technology, thyroid surgery has entered a precise era. Intraoperative nerve monitoring and intraoperative continuous nerve monitoring make the protection of recurrent laryngeal nerve more secure. Moreover, nanocarbon negative imaging technology could identify parathyroid gland more precise. However, when using nanocarbon, we should grasp the injection time, injection position and dosage to achieve the best effect of negative imaging. In the present study, we aimed to investigate the perioperative and outcomes of the application of nanocarbon negative imaging technology in the fifth stage of chronic kidney disease with SHPT, with a comparison to the control group without nanocarbon injection, so as to analyze the safety and efficacy of nanocarbon negative imaging technology in thyroid cancer surgery. We present the following article in accordance with the STROBE reporting checklist (available at https:// dx.doi.org/10.21037/gs-21-385).

\section{Methods}

\section{General information}

From January 2018 to August 2020, 89 patients with CDK5 and SHPT were enrolled in the study. Patients were divided into two groups according to whether or not they received nanocarbon suspension during the operation. The observation group (A) was composed of 46 patients including 24 males and 22 females, while in the control group (B) there were 43 patients, including 22 males and 21 females. There was no significant difference in age and duration of hemodialysis between the two groups $(\mathrm{P}>0.05)$. All procedures performed in this study involving human participants were in accordance with the Declaration of Helsinki (as revised in 2013). This study was approved by the Ethics the Committee of the Affiliated Hospital of Nantong University (ID: 2013-67). Informed consent was provided by all participants.

\section{Inclusion criteria}

The guidelines of the American Society of Nephropathy Foundation [2017] and the consensus of clinical practice experts of the Thyroid Surgeons Committee of the Surgeons Branch of the Chinese Medical Doctor Association [2016] (7) were combined to define the inclusion criteria, which were as follows:

(I) CKD5 patients with SHPT who had no history of neck surgery, such as thyroid or parathyroid surgery.

(II) If iPTH $>600-800 \mathrm{pg} / \mathrm{mL}$ or $\mathrm{iPTH}>450 \mathrm{pg} / \mathrm{mL}$ for more than two months, the decrease of iPTH was less than $50 \%$.

(III) Clinical manifestations included progressive ectopic calcification of the cardiovascular and cerebrovascular systems, severe bone pain, osteoporosis, myalgia, skin pruritus, and other symptoms that affect the quality of life.

(IV) After conservative treatment, blood calcium $>4 \mathrm{mmol} / \mathrm{L}$, and blood phosphorus $>1.94 \mathrm{mmol} / \mathrm{L}$.

(V) Ultrasound or CT examination showed that at least one parathyroid gland was enlarged with diameter $>1 \mathrm{~cm}$ or maximum volume $>300 \mathrm{~mm}^{3}$ or ${ }^{99 \mathrm{~m}} \mathrm{Tc}-$ MIBI showed high-density concentrated shadow.

(VI) Parathyroid glands in the chest were excluded before the operation.

(VII) There were no contraindications such as cardiopulmonary insufficiency and coagulation dysfunction.

\section{Operation method}

Total parathyroidectomy (tPTX) was performed using an arc-shaped collar incision in the anterior neck, about $5 \mathrm{~cm}$ in length. The platysma muscle was opened, and the free flap was placed under it, up to the thyroid cartilage and down to the supraclavicular fossa. The white line of the neck was cut along the median and the anterior cervical muscle was opened to expose the thyroid gland. At this time, group $B$ patients received a $0.15 \mathrm{~mL}$ of nanocarbon suspension injection into the left and right sides of the thyroid surface, and when drawing back, avoiding injection of blood. After the injection, local gauze was pressed for $1 \mathrm{~min}$, the middle thyroid vein was cut off, and the lateral space of the thyroid gland was freed. The posterior recurrent nerve was then exposed, and the parathyroid gland was located (Figure 1). At this time, bilateral thyroid and corresponding lymph 

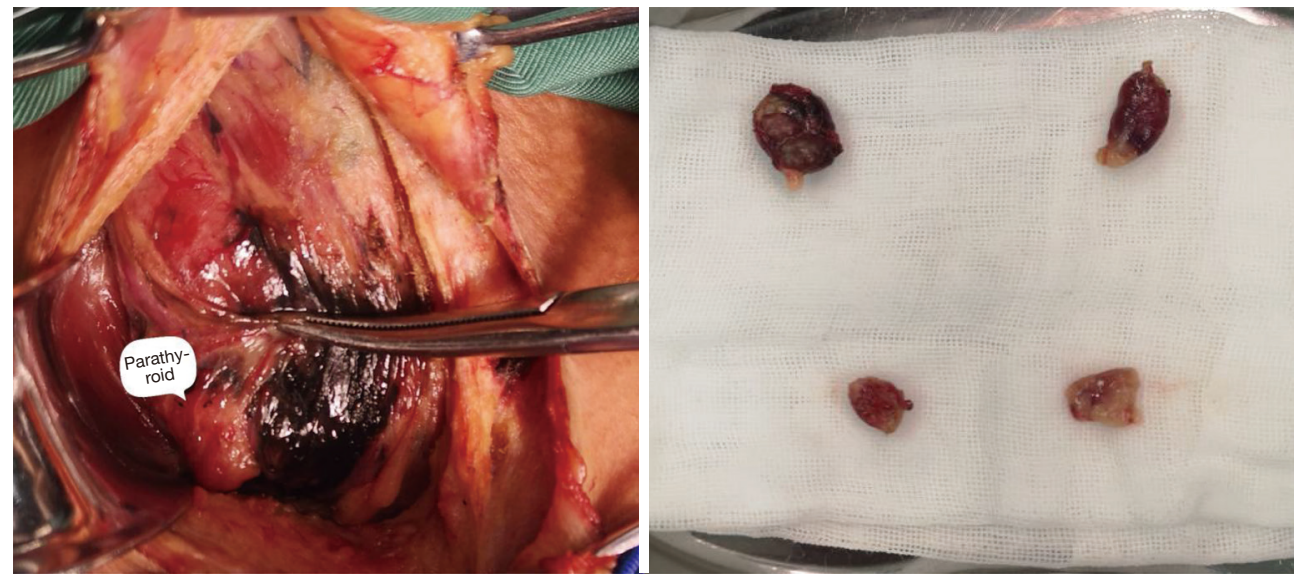

Figure 1 Exposure of the recurrent laryngeal nerve.

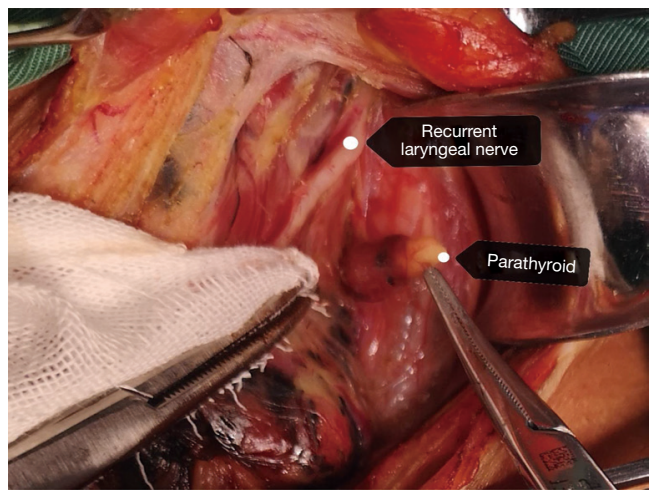

oral calcium supplements and calcitriol. General indexes included operation time ( $\mathrm{min})$, blood loss $(\mathrm{mL})$, intravenous calcium time (day), and bone pain relief time (day). Routine examination, blood ALP, iPTH, complete set of blood electrolytes, and pathological diagnosis were performed at six hours, 1 day, and 1 week after surgery then at 1 month and 6 months.

\section{Statistical analysis}

SPSS 22.0 statistical software was used to establish the database for statistical analysis. The measurement data were expressed by $(x \pm s)$ and the count data were expressed by rate (\%). All enumeration data were examined using the chi-squared test or $t$-test and $\mathrm{P}<0.05$ indicated a difference in statistical significance.

not, and appeared light yellow or light pink, with a clear position. The parathyroid glands were removed one by one without damaging the parathyroid capsule (Figure 2), and after the operation, a silicone drainage tube was placed in the neck.

\section{Observation index and follow-up}

Postoperative general anesthesia nursing and thyroid surgery routine nursing were implemented, as well as a bedside organ incision package. Routine hemodialysis without heparin was performed within one week to increase the frequency of hemodialysis. Postoperative management consisted of oral and intravenous calcium supplements $(10 \%$ calcium gluconate $10 \mathrm{~g}$ ) combined with intravenous injection of the micropump, and about one week after surgery, patients were gradually transitioned to

\section{Results}

\section{Comparison of perioperative indexes between groups $A$ and $B$}

The general clinical symptoms of bone pain, myalgia, and pruritus in the observation group were relieved (100\%), while in the control group, three cases had no significant remission (93.48\%). The operation time, blood loss, and bone pain relief time of the observation group were better than those of the control group $(\mathrm{P}<0.05)$, but there was no difference in the time of intravenous calcium between the two groups $(\mathrm{P}>0.05)$. In terms of complications, there were three cases of transient hoarseness and one of transient drinking cough in the control group which were relieved 
Table 1 Comparison of perioperative indexes between two methods $(\bar{x} \pm \mathrm{s})$

\begin{tabular}{lccccc}
\hline Group & Number of cases & Operation time $(\mathrm{min})$ & Blood loss $(\mathrm{mL})$ & Bone pain relief time $(\mathrm{d})$ & Intravenous calcium time $(\mathrm{d})$ \\
\hline A & 46 & $60.07 \pm 14.71$ & $23.04 \pm 10.6$ & $2.5 \pm 1.72$ & $5.93 \pm 0.93$ \\
B & 43 & $46.12 \pm 13.29$ & $10.1 \pm 6.7$ & $2.12 \pm 0.59$ & $5.84 \pm 0.10$ \\
\hline
\end{tabular}

Table 2 Two methods of blood iPTH monitoring $\left(\bar{x}_{ \pm}\right)$

\begin{tabular}{lccccc}
\hline Group & $\begin{array}{c}\text { Before treatment } \\
(\mathrm{pg} / \mathrm{mL})\end{array}$ & $\begin{array}{c}\text { Postoperative 1D } \\
(\mathrm{pg} / \mathrm{mL})\end{array}$ & $\begin{array}{c}1 \text { week after operation } \\
(\mathrm{pg} / \mathrm{mL})\end{array}$ & $\begin{array}{c}1 \text { month after the } \\
\text { operation }(\mathrm{pg} / \mathrm{mL})\end{array}$ & $\begin{array}{c}6 \mathrm{months} \text { after the } \\
\text { operation }(\mathrm{pg} / \mathrm{mL})\end{array}$ \\
\hline A & $1,903.41 \pm 638.31$ & $18.13 \pm 11.78$ & $19.20 \pm 9.50$ & $41.57 \pm 109.53$ & $46.80 \pm 131.93$ \\
B & $1,926.26 \pm 697.82$ & $13.16 \pm 4.28$ & $11.77 \pm 3.64$ & $12.91 \pm 4.26$ & $13.72 \pm 5.98$ \\
$\mathrm{P}$ & 0.87 & $<0.01$ & $<0.01$ & 0.02 & 0.03 \\
\hline
\end{tabular}

iPTH, intact parathyroid hormone level.

Table 3 Two methods were used to monitor blood calcium levels $(\bar{x} \pm \mathrm{s}, \mathrm{mmol} / \mathrm{L})$ in multiple groups

\begin{tabular}{lccccc}
\hline Group & $\begin{array}{c}\text { Before treatment } \\
(\mathrm{mmol} / \mathrm{L})\end{array}$ & $\begin{array}{c}6 \mathrm{~h} \text { after operation } \\
(\mathrm{mmol} / \mathrm{L})\end{array}$ & $\begin{array}{c}1 \text { week after operation } \\
(\mathrm{mmol} / \mathrm{L})\end{array}$ & $\begin{array}{c}1 \text { month after operation } \\
(\mathrm{mmol} / \mathrm{L})\end{array}$ & $\begin{array}{c}6 \text { months after operation } \\
(\mathrm{mmol} / \mathrm{L})\end{array}$ \\
\hline $\mathrm{A}$ & $2.68 \pm 0.34$ & $1.47 \pm 0.32$ & $1.62 \pm 0.36$ & $1.74 \pm 0.31$ & $1.95 \pm 0.42$ \\
$\mathrm{~B}$ & $2.49 \pm 0.28$ & $1.29 \pm 0.14$ & $1.48 \pm 0.19$ & $1.53 \pm 0.17$ & $1.73 \pm 0.22$ \\
\hline
\end{tabular}

W, week; M, month.

within a week of the use of neurotrophic drugs and aerosol inhalation of glucocorticoids. There were no serious complications such as permanent nerve injury or chylous leakage in both groups. The neck drainage was less than $5 \mathrm{~mL} / 24 \mathrm{~h}$ and was removed (Table 1 ).

\section{Comparison of blood iPTH monitoring level between groups $A$ and $B$}

A postoperative iPTH level greater than $150 \mathrm{pg} / \mathrm{mL}$ indicates recurrence, with a sensitivity of $100 \%$ and specificity of $99.5 \%$ (8). Our results showed that the recurrence rate of the observation group using nanocarbon was lower, and the difference was statistically significant $(\mathrm{P}<0.05)$. The exact value of tPTX in SHPT was reconfirmed in both groups of surgical results compared with pre-treatment results (Table 2).

\section{Comparison of blood calcium monitoring level between groups $A$ and $B$}

With a sudden decrease in iPTH levels, bone rapidly absorbs calcium, and the time window of calcium starvation is within 18 hours (9), manifesting as numbness around the mouth, numbness of limbs, and convulsions (10). We infused calcium gluconate by an intravenous micropump after surgery to account for this, and the dosage was adjusted in each patient. However, calcium could be taken orally on the first day after the operation, and the intravenous calcium was withdrawn about one week after surgery. The calcium level of the observation group was lower than the control group and the difference was statistically significant $(\mathrm{P}<0.05)$. The actual tPTX operation is more prone to hypocalcemia, but with the extension of time, the calcium level can gradually return to the normal level under the condition that only oral calcium is used. In the control group, the overall serum calcium level was higher than that in the observation group because of recurrent cases (Table 3).

\section{Discussion}

The choice of operation for patients with SHPT has always been the focus of controversy. The causes of postoperative recurrence of hyperparathyroidism are surgical omission, incomplete resection, or local implantation, which leads 
to long-term recurrence (11). Subtotal parathyroidectomy (SPTX) has a high recurrence rate, dangerous complications, and high mortality and is seldom used in the clinic. While the 2016 version of the China consensus has not yet been released, total parathyroidectomy plus autotransplantation $($ tPTX + AT) is a better choice for patients who may undergo renal transplantation in the future as it is easy and less difficult to select the smaller transplantation site for local resection. Recurrent, progressive SHPT is caused by hyperthyroidism at the transplantation site or parathyroid tissue missed at first surgery, and it is very important to locate its source. For patients without a renal transplantation plan, the recurrence rate of total parathyroidectomy (tPTX) is lower than that of tPTX + AT (12). Although the onset and degree of hypocalcemia within six months after surgery are higher than those of tPTX + AT, tPTX produces better outcomes in the incidence of bone and mineral metabolism disorders $(13,14)$ and bone density. tPTX can reduce the level of iPTH, improve disorders of calcium and phosphorus metabolism, and relieve the clinical symptoms caused by a series of lesions in the skin, soft tissue, bone, and blood vessels. It can also even improve sleep quality, mental health, behavioral health, quality of life, and social function $(12,15)$.

There are two major benefits to the use of tPTX over other methods. Firstly, CDK5 patients have overall poor health and are at greater risk of complications from any procedure involving general anesthesia. The decision to remove all parathyroid tissue at the first operation decreases the likelihood that further procedures are required. Secondly, the objective of fully removing all tissue at first operation renders the identification of the anatomical boundaries for surgery more precise, increasing the likelihood of its success and also reduces the need for further procedures. Reoccurrence is more likely following partial removal as residual tissue may be missed, and a second operation significantly increases the incidence of damage to nerves, blood vessels, and the thoracic duct (16). To sum up, minimizing the recurrence rate and avoiding the need for subsequent surgery offers the best outcome for uremic patients with SHPT.

The use of B-ultrasound and enhanced CT examination for preoperative positioning only provides surgeons with a general impression of the parathyroid gland, and even MIBI-SPECT/CT has a high rate of missed detection (about $>20 \%$ ) (17). ${ }^{99 \mathrm{~m}} \mathrm{Tc}-$ MIBI preoperative injection intraoperative positioning $\gamma$ detectors are not available in every hospital, and as each operation requires consultation with nuclear medicine doctors to prepare for surgery it is not very convenient (18). As a safe tracer, nanocarbon is widely used in lymph node dissection for thyroid cancer. After injection, the tracer quickly gathers in the lymph nodes and thyroid tissue turning them black, as it passes through lymphatic vessels and not through capillaries. This greatly benefits surgeons by protecting important tissues such as the posterior recurrent laryngeal nerve, parathyroid gland and local lymph nodes (19). In this study, there was no recurrence, nerve injury, chylous leakage, or other complications in the nanocarbon operation group during the observation period. This method has obvious advantages in improving the operation quality, shortening the operation time, and improving the speed of relieving clinical symptoms such as bone pain. During surgery for ectopic parathyroid tissue, we followed the procedure of opening the dorsal side of the thyroid gland, exploring around the esophagus, central group lymph nodes, thymus, and cervical vascular sheath. In the observation group, the lymph nodes were displayed by the injection of nanocarbon and it was easy to distinguish tissue which stained black for that which did not. For a small number of ectopic parathyroid glands that were not deep in the thyroid gland, the circular or oval part of the thyroid capsule could be found without black stain, and when the thyroid gland was opened, the ectopic parathyroid glands were found. At the same time, the nerve was displayed and could be easily avoided. During the operation, the parathyroid capsule was not damaged, which allowed implantation in the operative area to be avoided and reduced the long-term recurrence rate. If the number of parathyroid glands is less than four through rapid intraoperative freezing, intraoperative parathyroid hormone assay (IOPTH) can be performed and if the IOPTH is still more than $150 \mathrm{pg} / \mathrm{mL}$ in 20 minutes, or the decrease rate is less than $80 \%$, it is likely there are still suspicious lesions (20). However, if combined with preoperative imaging localization, the fourth parathyroid gland cannot be found by intraoperative reexploration, we believe thymectomy should be employed. According to our follow-up results, the outcome of the group receiving nanocarbon was better than those not receiving it 6 months after surgery. There is no universal method for the surgical treatment of SHPT and individual variations must be considered. Preoperative localization is a very important step in determining the minimum number and location of surgical resection. Studies with a larger sample size and longer follow-up are required to determine long-term survival rates, cardiovascular outcomes, and other indicators. 


\section{Conclusions}

Nanocarbon negative imaging technology in total parathyroidectomy can improve the surgical effect, reduce the risk of treatment, and improve the prognosis of patients.

\section{Acknowledgments}

Funding: This work was supported by grants from the Research Project of Maternal and Child Health of Jiangsu Province (F201953), the Science and Technology Project of Nantong (JC2020067) to ZH, and the Research project of Nantong Health and Family Planning Commission (QA2019002) to YJ.

\section{Footnote}

Reporting Checklist: The authors have completed the STROBE reporting checklist. Available at https://dx.doi. org/10.21037/gs-21-385

Data Sharing Statement: Available at https://dx.doi. org/10.21037/gs-21-385

Conflicts of Interest: All authors have completed the ICMJE uniform disclosure form (available at https://dx.doi. org/10.21037/gs-21-385). The authors have no conflicts of interest to declare.

Ethical Statement: The authors are accountable for all aspects of the work in ensuring that questions related to the accuracy or integrity of any part of the work are appropriately investigated and resolved. All procedures performed in this study involving human participants were in accordance with the Declaration of Helsinki (as revised in 2013). This study was approved by the Ethics the Committee of the Affiliated Hospital of Nantong University (ID: 2013-67). Informed consent was provided by all participants.

Open Access Statement: This is an Open Access article distributed in accordance with the Creative Commons Attribution-NonCommercial-NoDerivs 4.0 International License (CC BY-NC-ND 4.0), which permits the noncommercial replication and distribution of the article with the strict proviso that no changes or edits are made and the original work is properly cited (including links to both the formal publication through the relevant DOI and the license).
See: https://creativecommons.org/licenses/by-nc-nd/4.0/.

\section{References}

1. 1. Galassi A, Ciceri P, Fasulo E, et al. Management of Secondary Hyperparathyroidism in Chronic Kidney Disease: A Focus on the Elderly. Drugs Aging 2019;36:885-95.

2. Chen TK, Knicely DH, Grams ME. Chronic Kidney Disease Diagnosis and Management: A Review. JAMA 2019;322:1294-304.

3. Iglesias P, Díez JJ. Endocrine Complications of Surgical Treatment of Thyroid Cancer: An Update. Exp Clin Endocrinol Diabetes 2017;125:497-505.

4. Shen Q, Xiang W, Ye S, et al. Plasma metabolite biomarkers related to secondary hyperparathyroidism and parathyroid hormone. J Cell Biochem 2019;120:15766-75.

5. Yu Y, Diao Z, Wang Y, et al. Hemodialysis patients with low serum parathyroid hormone levels have a poorer prognosis than those with secondary hyperparathyroidism. Ther Adv Endocrinol Metab 2020;11:2042018820958322.

6. van der Plas W, Kruijff S, Sidhu SB, et al. Parathyroidectomy for patients with secondary hyperparathyroidism in a changing landscape for the management of end-stage renal disease. Surgery 2021;169:275-81.

7. Isakova T, Nickolas TL, Denburg M, et al. KDOQI US Commentary on the 2017 KDIGO Clinical Practice Guideline Update for the Diagnosis, Evaluation, Prevention, and Treatment of Chronic Kidney DiseaseMineral and Bone Disorder (CKD-MBD). Am J Kidney Dis 2017;70:737-51.

8. Zhang L, Xing C, Shen C, et al. Diagnostic Accuracy Study of Intraoperative and Perioperative Serum Intact PTH Level for Successful Parathyroidectomy in 501 Secondary Hyperparathyroidism Patients. Sci Rep 2016;6:26841.

9. Wang M, Chen B, Zou X, et al. A Nomogram to Predict Hungry Bone Syndrome After Parathyroidectomy in Patients With Secondary Hyperparathyroidism. J Surg Res 2020;255:33-41.

10. Delos Santos R, Rossi A, Coyne D, et al. Management of Post-transplant Hyperparathyroidism and Bone Disease. Drugs 2019;79:501-13.

11. Kose E, Rudin AV, Kahramangil B, et al. Autofluorescence imaging of parathyroid glands: An assessment of potential indications. Surgery 2020;167:173-9.

12. Silveira AA, Brescia MDG, do Nascimento CP Jr, et al. 
Critical analysis of the intraoperative parathyroid hormone decrease during parathyroidectomy for secondary and tertiary hyperparathyroidism. Surgery 2020;168:1079-85.

13. Schlosser K, Bartsch DK, Diener MK, et al. Total Parathyroidectomy With Routine Thymectomy and Autotransplantation Versus Total Parathyroidectomy Alone for Secondary Hyperparathyroidism: Results of a Nonconfirmatory Multicenter Prospective Randomized Controlled Pilot Trial. Ann Surg 2016;264:745-53.

14. Fang L, Wu J, Luo J, et al. Changes in bone mineral density after total parathyroidectomy without autotransplantation in the end-stage renal disease patients with secondary hyperparathyroidism. BMC Nephrol 2018;19:142.

15. Lau WL, Obi Y. Parathyroidectomy in the Management of Secondary Hyperparathyroidism. Clin J Am Soc Nephrol 2018;13:952-61.

16. Zhu L, Cheng F, Zhu X, et al. Safety and effectiveness of reoperation for persistent or recurrent drug refractory secondary hyperparathyroidism. Gland Surg 2020;9:401-8.

17. Yang J, Wang H, Zhang J, et al. Sestamibi Single-

Cite this article as: Wu Y, Liu Y, Huang T, Jiang Y, Wang H, He Z. Application of nanocarbon negative imaging technology in surgery for secondary hyperparathyroidism. Gland Surg 2021;10(8):2455-2461. doi: 10.21037/gs-21-385
Positron Emission Computed Tomography/Diagnosticquality Computed Tomography for the localization of abnormal parathyroid glands in patients with primary hyperparathyroidism: What clinicopathologic factors affect its accuracy? J Endocrinol Invest 2021. doi: 10.1007/ s40618-020-01471-7.

18. Sun HM, Chen F, Yin HL, et al. Rapid development of metastatic pulmonary calcifications in primary hyperparathyroidism: a case report and literature review. Diagn Pathol 2017;12:38.

19. Yan S, Zhao W, Wang B. A Novel Technology for Localization of Parathyroid Adenoma: UltrasoundGuided Fine Needle Aspiration Combined With Rapid Parathyroid Hormone Detection and Nano-Carbon Technology. Surg Innov 2018;25:357-63.

20. Lang BH, Fung MMH. Intraoperative parathyroid hormone (IOPTH) assay might be better than the secondgeneration assay in parathyroidectomy for primary hyperparathyroidism. Surgery 2021;169:109-13.

(English Language Editor: B. Draper) 